\title{
Tracing the base of protostellar wind(s) towards the high-mass star forming region AFGL 5142: VLA continuum and VLBA $\mathrm{H}_{2} \mathrm{O}$ maser observations
}

\author{
C. Goddi ${ }^{1,2}$ and L. Moscadelli ${ }^{1}$ \\ 1 INAF, Osservatorio Astronomico di Cagliari, Loc. Poggio dei Pini, Str. 54, 09012 Capoterra (CA), Italy \\ e-mail: cgoddi@ca.astro.it \\ 2 Dipartimento di Fisica, Università degli Studi di Cagliari, S.P. Monserrato-Sestu Km 0.7, 09042 Cagliari, Italy
}

Received 28 June 2005 / Accepted 21 September 2005

\begin{abstract}
We have conducted phase-reference multi-epoch observations of the $22.2 \mathrm{GHz}$ water masers using the Very Long Baseline Array (VLBA) and a multi-frequency study of the continuum emission using the Very Large Array (VLA) towards the high-mass star forming region (SFR) AFGL 5142. 29 maser features were identified and most of them were persistent over the four observing epochs, allowing absolute proper motions to be determined. The water maser emission comes from two elongated structures (indicated as Group I and Group II), with the measured proper motions aligned along the structures' elongation axes. Each group consists of two (blue- and red-shifted) clusters of features separated by a few hundreds and thousands of AU respectively for Group I and Group II.

The maser features of Group II have both positions and velocities aligned along a direction close to the axis of the outflow traced by $\mathrm{HCO}^{+}$and $\mathrm{SiO}$ emission on angular scales of tens of arcsec. We predict that the maser emission arises from dense, shocked molecular clumps displaced along the axis of the molecular outflow. The two maser clusters of Group I are oriented on the sky along a direction forming a large angle $\left(\gtrsim 60^{\circ}\right)$ with the axis of the jet/outflow traced by Group II maser features. We have detected a compact (8.4 and $\left.22 \mathrm{GHz}\right)$ continuum source (previously reported at 4.9 and $8.4 \mathrm{GHz}$ ) that falls close to the centroid of Group I masers, indicating that the source ionizing the gas is also responsible for the excitation of the water masers. The kinematic analysis indicates that the Group I masers trace outflowing rather than rotating gas, discarding the Keplerian disk scenario proposed in a previous paper for Group I. Since the axis joining the two maser clusters of Group II does not cross the position of the continuum source, Group II masers might be excited by an (undetected) massive YSO, distinct from the one (pinpointed by the VLA continuum emission) responsible for the excitation of the Group I masers.

Our results give support to models of accretion and jet ejection related to the formation of high-mass stars.
\end{abstract}

Key words. masers - stars: formation - ISM: kinematics and dynamics - ISM: jets and outflows - accretion, accretion disks radio continuum: ISM

\section{Introduction}

A widely accepted, observationally supported, "standard" theory exists for the formation of low-mass, solar-type stars, involving inside-out collapse of molecular cloud fragments and formation of accretion disks (e.g. Shu et al. 1987). For highmass $\left(\gtrsim 10 M_{\odot}\right)$ stars, on the contrary, further effort (from both a theoretical and observational point of view) is required to improve our understanding of their formation process. A fundamental question is whether the mode of high-mass formation is a scaled-up version of the low-mass forming mode or whether it involves different physical processes. This question arises because stars with masses larger than $8 M_{\odot}$ contract to the main sequence and ignite while still accreting matter from the infalling envelope. Then, the "standard" theory predicts that, for a spherically symmetrical collapse, the radiation pressure produced by the massive YSO enormous luminosity would be sufficient to inhibit any further accretion. That leads to the paradox that massive stars $\left(M>8 M_{\odot}\right)$ might never form, in disagreement with the observed upper limits of hundreds of solar masses. In order to circumvent this dilemma, it has been proposed that high-mass stars might build up via merging of lower mass $\left(M<8 M_{\odot}\right)$ YSOs (coalescence model - e.g. Bonnell \& Bate 2002), or, alternatively, by accreting material from the infalling envelope via a thin disk, as it occurs in lowmass stars (accretion model - e.g. Yorke \& Sonnhalter 2002). In the disk scenario, in order to allow the gas of the disk to accrete onto the stellar surface, the removal of the excess angular momentum is thought to be associated with the ejection of a powerful bipolar outflow, collimated along the disk-axis. Then the accretion paradigm would predict the occurrence of disk/outflow systems, whereas the alternative coalescence scenario would imply the destruction of ordered kinematical structures surrounding the merging protostars.

According to widely accepted theories, (proto)stellar jets may be launched by the combined action of magnetic and 
centrifugal forces along the magnetic field lines threading the YSO's envelope. The magnetohydrodynamical (MHD) models proposed to explain the origin of (proto)stellar jets can be divided into two categories, depending on the place where the jet is supposed to originate: 1) $X$-winds, which are magnetized stellar winds driven by the stellar magnetic field, arising at the star/disk boundary (see Shu et al. 2000, for a review); 2) diskwinds, where the jet stems from the surface of a Keplerian disk threaded by the circumstellar magnetic field (Konigl \& Pudritz 2000, and references therein).

A possible way, from an observational point of view, to distinguish between alternative (accretion and jet ejection) models in massive star formation theories is by studying the kinematics of the gas in the proximity $(<100 \mathrm{AU})$ of the YSO, corresponding to the "root" of the putative jet/disk system. An ideal diagnostic tool is provided by Very Long Baseline Interferometry (VLBI) observations at centimeter $(\mathrm{cm})$ wavelengths of maser transitions of molecular species (such as $\mathrm{OH}$, $\mathrm{H}_{2} \mathrm{O}, \mathrm{CH}_{3} \mathrm{OH}$ ). In particular, for fast-moving $22.2 \mathrm{GHz}$ water masers, multi-epoch VLBI observations, reaching angular resolutions of $\simeq 0.5$ mas (corresponding to $\simeq 1 \mathrm{AU}$ at a distance of $2 \mathrm{kpc}$ ), permit accurate proper motions to be determined with time baselines as short as a few months. Combining the measured tangential velocities with the radial velocities derived via the Doppler effect, allows one to obtain the 3D velocity distribution of the masing gas.

Previous VLBI observations have shown that $22.2 \mathrm{GHz}$ water masers are preferentially associated with collimated (jets) or wide-angle flows of gas at the base of larger-scale molecular outflows (W3 IRS 5, Imai et al. 2000; W75N-VLA1, Torrelles et al. 2003; Sh 2-255 IR, IRAS 23139+5939, WB89-234, and OMC2, Goddi et al. 2005; IRAS 20126+4104, Moscadelli et al. 2005). In a few objects (NGC 2071 IRS $1 / 3$, Seth et al. 2002; AFGL 5142, Goddi et al. 2004 - hereafter Paper I), accretion disks have been proposed to explain linear clusters (size $\sim 10-100 \mathrm{AU}$ ) of $\mathrm{H}_{2} \mathrm{O}$ masers detected through VLBI observations.

The $22.2 \mathrm{GHz} \mathrm{H}_{2} \mathrm{O}$ masers in AFGL 5142 (for a detailed description of the source see Paper I) were observed with the European VLBI Network (EVN) at four epochs (from October 1996 to November 1997) and found to be distributed across two structures (indicated as "Group I" and "Group II"). Such structures have been interpreted as being possibly associated with a disk/jet system, indicating that AFGL 5142 may be an ideal candidate to test the formation theories of high-mass stars. However, the proposed interpretation needs more stringent observational constraints, the multiepoch EVN observations suffering two major drawbacks: 1) too long time separation between consecutive epochs ( $\geq 3$ months, more than the average life time of the maser features); 2) limited sensitivity (average detection threshold of $\sim 0.3 \mathrm{Jy}_{\text {beam }}{ }^{-1}$ ). These limitations prevented us from detecting and measuring the proper motions of the weakest and less-longeval maser features.

Recently, we performed follow-up observations of the $22 \mathrm{GHz} \mathrm{H}_{2} \mathrm{O}$ masers in AFGL 5142 using the Very Long Baseline Array (VLBA), taking advantage of both high sensitivity and an optimized time separation between consecutive epochs. The VLBA observations were performed in phase-reference mode to derive the absolute positions and proper motions of the water maser features.

We have also performed multi-frequency VLA-B continuum observations of AFGL 5142, aiming to precisely locate the position of the exciting $\mathrm{YSO}(\mathrm{s})$, to put constraints on the nature of the previously observed radio continuum emission (Carpenter et al. 1990; McCutcheon et al. 1991; Torrelles et al. 1992; Hunter et al. 1995, 1999; Carral et al. 1999), and, finally, to investigate the relationship between the water masers and the continuum source.

Section 2 describes our VLA and multi-epoch VLBA observations and gives technical details on the data analysis. Section 3 compares our results with previous interferometrical observations of the region. In Sect. 4, plausible kinematic models for interpreting the measured positions and velocities of the maser features are investigated. Conclusions are drawn in Sect. 5.

\section{Observations and data analysis}

\subsection{VLA observations}

The continuum observations were performed using the VLA in its B configuration on March 1 and 11, 2005, for a total observing time of $4 \mathrm{~h}$. We observed a bandwidth of $50 \mathrm{MHz}$ centered at a central frequency of $8.4601,22.4601$, and $43.3399 \mathrm{GHz}(X$, $K$, and $Q$-bands, respectively). The data were edited, calibrated, and imaged using the NRAO's Astronomical Image Processing System (AIPS) package. Absolute amplitude calibration was obtained from observations of 3C 147 (with flux density of 4.8, 1.8 , and $0.9 \mathrm{Jy}$ for $X, K$, and $Q$-band, respectively). In each band, the observations were performed using the "fast switching" method, where short (420, 180, 60 s) scans of AFGL 5142 were alternated with $60 \mathrm{~s}$ scans of the nearby phase calibrator J05181+33062. The error in the absolute calibration is estimated to be within $10 \%$ in $X, 20 \%$ in $K$ and $30 \%$ in $Q$-band. Table 1 reports the main observational parameters (restoring beam $F W H M$ sizes and position angle, and the map rms noise level) for the three observing bands.

\subsection{VLBA observations}

\subsubsection{VLBA runs}

The observations in the $6_{16}-5_{23} \mathrm{H}_{2} \mathrm{O}$ maser line at $22.2 \mathrm{GHz}$ were conducted using the VLBA at four epochs (16 October and 22 November 2003, 1 January and 8 February 2004), each epoch lasting for $12 \mathrm{~h}$. The observations were performed in phase-reference mode, alternating scans on the maser source, AFGL 5142, and the phase-reference source, J0518+3306, with a switching cycle of $70 \mathrm{~s}$. J05181+33062 is separated $2.6^{\circ}$ from AFGL 5142, belongs to the VLBA calibrator catalog, and has very accurate coordinates (RA and Dec uncertainties $<1$ mas). Interlapsed every $\approx 80 \mathrm{~min}, 3$-min scans on several continuum sources $(0528+134,0552+398,0333+321$, $0642+449)$ were observed for calibration purposes. The total on-source integration time for the maser target was about $5.5 \mathrm{~h}$.

Both circular polarizations were recorded using a $16 \mathrm{MHz}$ bandwidth centered on the LSR velocity of $-4.8 \mathrm{~km} \mathrm{~s}^{-1}$. The 
Table 1. Radio continuum observations.

\begin{tabular}{ccccccccc}
\hline \hline Band & $\begin{array}{c}\text { Beam size } \\
\left({ }^{\prime \prime}\right)\end{array}$ & $\begin{array}{c}\text { Beam PA } \\
\left({ }^{\circ}\right)\end{array}$ & $\begin{array}{c}\sigma \\
\left(\mu \mathrm{Jy} \mathrm{beam}^{-1}\right)\end{array}$ & $\begin{array}{c}\alpha(\mathrm{J} 2000) \\
(\mathrm{h} \mathrm{m} \mathrm{s})\end{array}$ & $\begin{array}{c}\delta(\mathrm{J} 2000) \\
\left({ }^{\circ} \prime^{\prime \prime}\right)\end{array}$ & $\begin{array}{c}S_{v} \\
(\mathrm{mJy})\end{array}$ & $\begin{array}{c}F W H M \text { Size } \\
(\operatorname{arcsec})\end{array}$ & $\begin{array}{c}\text { PA } \\
\left({ }^{\circ}\right)\end{array}$ \\
\hline$X$ & $0.88 \times 0.82$ & 71 & 26 & 053048.019 & 334754.6 & $0.75 \pm 0.08^{a}$ & - & - \\
$K$ & $0.38 \times 0.38$ & 0 & 52 & 053048.020 & 334754.5 & $0.7 \pm 0.1$ & $0.35 \times 0.27^{b}$ & 130 \\
$Q$ & $0.17 \times 0.15$ & 31 & 270 & - & - & $<0.9^{c}$ & - & - \\
\hline
\end{tabular}

Note. For each observing band, Cols. 2 and 3 give respectively the FWHM sizes along the major and minor axes, and the position angle of the restoring beam; Col. 4 the rms noise level on the maps; Cols. 5 and 6 the RA and Dec absolute position of the intensity peak; Col. 7 the integrated flux density of the maps; Cols. 8 and 9 the deconvolved FWHM sizes and the position angle of the detected sources.

${ }^{a}$ The absolute flux density uncertainty is estimated to be $\leq 10 \%$ at $X$-band, $\leq 20 \%$ at $K$-band, $\leq 30 \%$ at $Q$-band.

${ }^{b}$ The source is compact at all observed frequencies, with the exception of the $K$-band, for which the deconvolving routine (SAD) produced FWHM sizes comparable with the observing beam.

${ }^{c}$ At $43.3 \mathrm{GHz}$ the flux density upper limit corresponds to $3 \sigma$.

data were correlated with the VLBA FX correlator in Socorro (New Mexico) with an integration period of $1 \mathrm{~s}$. The correlator used 1024 spectral channels corresponding to a channel separation of $0.2 \mathrm{~km} \mathrm{~s}^{-1}$.

\subsubsection{Data reduction}

Data reduction was performed using the AIPS package, following the standard procedure for VLBI line data. Total power spectra of the continuum calibrators were used to derive the bandpass response of each antenna. The amplitude calibration was performed using the information on the system temperature and the gain curve of each antenna.

For each observing epoch, a single scan of a strong calibrator was used to derive the instrumental (time-independent) single-band delay and the phase offset between the two polarizations. After removing the instrumental errors, all calibrator scans were fringe-fitted to determine the residual (timedependent) delay and the fringe rate. The corrections derived from calibrators were applied to the strongest maser component, chosen to refer the visibilities of all the other maser emission channels. In each observing epoch the strongest maser component emitted at the same velocity, $V_{\mathrm{LSR}}=-4.9 \mathrm{~km} \mathrm{~s}^{-1}$, and exhibited a simple spatial structure consisting of a single, almost unresolved spot. The visibilities of the reference channel were fringe-fitted to find the residual fringe rate produced both by differences in atmospheric fluctuations between the calibrators and the maser, and by errors in the model used at the correlator. After correcting for the residual fringe rate, the visibilities of the reference channel were self-calibrated to remove any possible effect of spatial structure. Finally, the corrections derived from the reference channel were applied to data of all spectral channels.

Spectral channel maps were produced extending over a sky area of $(\Delta \alpha \cos \delta \times \Delta \delta) 6^{\prime \prime} \times 6^{\prime \prime}$ and covering the whole velocity range where signal was visible in the total-power spectra (from -10 to $5 \mathrm{~km} \mathrm{~s}^{-1}$ ). At each epoch, the maser emission centres are found to be distributed within an area of $2^{\prime \prime} \times 1$.' 5 . The CLEAN beam was an elliptical Gaussian with a $F W H M$ size of $0.7 \times 0.4$ mas. In each observing epoch, the rms noise level on the channel maps, $\sigma$, varied over a similar range of values, 4-40 mJy beam ${ }^{-1}$.

\subsubsection{Absolute position determination}

Auto-referencing the visibilities phases of the maser data causes the loss of the absolute position information. Since in our case the water maser source is significantly stronger than the phase reference source, J0518+3306, we first attempted to determine the maser absolute position using the reverse phase referencing technique. All the phase calibration (fringefitting + self-calibration) solutions obtained working on the reference maser component were applied to the J05181+33062 data. So doing, the position of the phase-reference source is shifted by a vector equal in absolute value but with opposite sign with respect to the offset of the maser reference spot from its correlated position. Finally, the maser-referenced visibilities of J05181+33062 were Fourier-transformed, but the resulting map produced only a marginal detection (with a peak flux density of $\sim 10 \mathrm{mJy}$ ).

Then, we experimented with an alternative way of direct phase-referencing, fringe-fitting the scans of J05181+33062 and applying the obtained phase calibration solutions to the maser data. The image constructed by Fourier transforming the visibilities of the maser source, calibrated with the phasereference continuum solutions, presented a signal-to-noise ratio sufficiently high to allow us to determine the offset of the reference maser (from the position used at the correlator) with an accuracy of tenths of milliarcsec. For the first observing epoch, the derived positional offset is: $\Delta \alpha_{1}=-0.0831 \pm$ $0.00035, \Delta \delta_{1}=-0.24469 \pm 0.0^{\prime} 0002$. The uncertainty on the positional offset is estimated by the ratio of the FWHM beam size to the dynamic range of the map. In order to obtain the peculiar motion of the maser source, the offsets derived at each epoch have been corrected for systematic effects associated to Galactic rotation, annual parallax and solar motion. The corrected offsets of the reference feature are reported in Table 2.

\subsubsection{Identification of maser emission}

Each channel map of the maser source was searched for emission above a detection threshold taken equal to the absolute value of the minimum in the map (varying across the channels in the range $5-7 \sigma)$. The detected maser spots were fitted with two-dimensional elliptical Gaussians, determining 


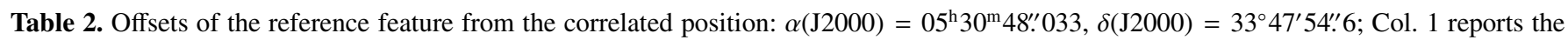
observing epoch; Cols. 2 and 3 respectively the RA and Dec apparent offsets, as derived from the phase-reference calibrated maps; Cols. 4 and 5 the corrections for the combined action of Galactic, Sun and earth motion; Cols. 6 and 7 the corrected (RA and Dec) offsets.

\begin{tabular}{ccccccc}
\hline \hline Epoch & $\begin{array}{c}\Delta \alpha_{1} \\
(\mathrm{mas})\end{array}$ & $\begin{array}{c}\Delta \delta_{1} \\
(\mathrm{mas})\end{array}$ & $\begin{array}{c}\Delta \alpha_{2} \\
(\mathrm{mas})\end{array}$ & $\begin{array}{c}\Delta \delta_{2} \\
(\mathrm{mas})\end{array}$ & $\begin{array}{c}\Delta \alpha_{f} \\
(\mathrm{mas})\end{array}$ & $\begin{array}{c}\Delta \delta_{f} \\
(\mathrm{mas})\end{array}$ \\
\hline 16 Oct. 03 & $-83.1(0.4)$ & $-244.7(0.2)$ & -1.2 & 1.0 & $-84.3(0.4)$ & $-243.7(0.2)$ \\
22 Nov. 03 & $-83.4(0.1)$ & $-244.6(0.1)$ & -0.9 & 1.2 & $-84.3(0.1)$ & $-243.4(0.1)$ \\
1 Jan. 04 & $-83.7(0.3)$ & $-245.3(0.2)$ & -0.6 & 1.4 & $-84.3(0.3)$ & $-243.9(0.2)$ \\
8 Feb. 04 & $-84.0(0.3)$ & $-245.5(0.2)$ & -0.3 & 1.7 & $-84.3(0.3)$ & $-243.8(0.2)$ \\
\hline
\end{tabular}

position, flux density, and FWHM size of the emission. Consistently to Paper I, the term of "feature" is used to indicate a collection of spectrally and spatially contiguous maser spots. A maser feature is considered real if it is detected in at least two contiguous channels (i.e., line-width $>0.4 \mathrm{~km} \mathrm{~s}^{-1}$ ), with a position shift of the intensity peak from channel to channel smaller than the FWHM size. The VLBA observations provided an improvement in sensitivity of about an order of magnitude respect to the previous EVN observations, lowering the detection threshold to $\sim 30 \mathrm{mJy}$ beam $^{-1}$ and allowing the detection of a much larger number of maser spots and features. In each observing epoch, about 270 maser spots were identified, grouped into 29 distinct maser features. Table 3 gives the parameters of the identified features. The absolute (RA and Dec) positional offsets of a given feature, evaluated with respect to the maser position used at the correlator $(\alpha(\mathrm{J} 2000)=$ $05^{\mathrm{h}} 30^{\mathrm{m}} 48^{\prime \prime}$. $033, \delta(\mathrm{J} 2000)=33^{\circ} 47^{\prime} 54^{\prime \prime}$ ' 6$)$, are calculated with a two step procedure. First, relative positions (respect to the reference maser spot) are derived from the (error-weighted) mean of the maser spot positions contributing to the feature emission. Then, at each epoch the relative positions are combined with the absolute positional offsets of the reference spot (reported in Table 2). For each feature, the absolute positional errors are estimated taking the sum of the feature relative positional uncertainties (evaluated by the weighted standard deviation of the spot positions) with the absolute positional uncertainties of the reference spot.

\subsubsection{Proper motion measurements}

For the features persistent over three or four epochs, the proper motions have been calculated performing a (error-weighted) linear least-squares fit of the positional offsets with time. For the VLBA observations the time separation between consecutive epochs was shorter than for the previous EVN observations ( $\sim 1$ month vs. 3-6 months) and that allowed to measure the proper motions of the most variable features, increasing significantly the number of measured proper motions ( $23 \mathrm{vs} .2$ of previous EVN observations, considering only features persistent over $\geq 3$ epochs). For a representative sample of persistent features, Fig. 1 shows the time variation of the RA and Dec (relative) offsets and the best linear fit giving the proper motion. Among the features observed at three or more epochs, the proper motions are derived only for those moving in a straight line at constant velocity (within the positional errors). Adopting such a criterion, no proper motion is derived for the feature with label number " 26 ", although detected at all the four observing epochs.

The derived absolute proper motions are reported in Table 3. The uncertainties of the absolute proper motions are the formal errors of the linear least-squares fits and are generally much larger than those associated with the relative proper motions, owing to the relatively large error of the absolute position of the reference spot. The phase-reference calibrator is too weak and too detached from the maser target to allow an effective removal of the atmospheric noise affecting the maser visibilities.

\section{Observational results}

\subsection{Radio continuum emission}

The VLA-B, $X$ and $K$-band continuum images of AFGL 5142, above a detection threshold of $5 \sigma$, show a single, compact source coinciding (within the positional errors) with the previously detected emission at $4.9 \mathrm{GHz}$ (Carpenter et al. 1990; McCutcheon et al. 1991) and 8.4 GHz (Torrelles et al. 1992; Hunter et al. 1995, 1999; Carral et al. 1999). No emission was detected at $43 \mathrm{GHz}$ and only a $3 \sigma$ upper limit to the source flux density could be derived. Table 1 gives the measured positions and flux densities for the three observing bands. At $K$-band, the emission appears slightly resolved, with a deconvolved elliptical Gaussian shape having a FWHM size (estimated using the AIPS task SAD) of $0.35 \times 0.27$ (corresponding to a diameter of $\sim 600 \mathrm{AU}$ ), and elongated along a southeast-northwest (SE-NW) direction.

Previous observations at $8.4 \mathrm{GHz}$ continuum, conducted using the VLA in A (Hunter et al. 1995), B (Hunter et al. 1999), C (Torrelles et al. 1992), and D (Carral et al. 1999) configurations, all show a compact continuum emission, with the exception of Torrelles et al. (1992), who presented marginal indication for the emission being extended at scales of $\sim 20^{\prime \prime} 1$ (see Fig. 3 of Torrelles et al. 1992). Figure 2 reports our and previous continuum observations towards AFGL 5142. At 8.4 GHz we have reported only our VLA-B measurement, not considering the previous value of Hunter et al. (1999), which is much more uncertain ${ }^{2}$. At both 4.9 and $8.4 \mathrm{GHz}$, the

\footnotetext{
1 In the Torrelles et al. (1992) map, the presence of extended emission is suggested only by the weakest contours, below the $3 \sigma$ level.

2 The $8.4 \mathrm{GHz}$ observations were conducted by Hunter et al. (1999) in snapshot mode, with a few minutes of integration, whereas our 8.4 GHz observations last for about one hour, allowing a significant sensitivity improvement.
} 
Table 3. Parameters of maser features detected with the VLBA in AFGL 5142.

\begin{tabular}{|c|c|c|c|c|c|c|c|c|}
\hline & Feature & $\begin{array}{c}V_{\mathrm{LSR}} \\
\left(\mathrm{km} \mathrm{s}^{-1}\right)\end{array}$ & $\begin{array}{l}F_{\text {int }} \\
(\mathrm{Jy}) \\
\end{array}$ & $\begin{array}{c}\Delta \alpha \\
\text { (mas) }\end{array}$ & $\begin{array}{c}\Delta \delta \\
(\mathrm{mas})\end{array}$ & $\begin{array}{c}V_{x} \\
\left(\mathrm{~km} \mathrm{~s}^{-1}\right)\end{array}$ & $\begin{array}{c}V_{y} \\
\left(\mathrm{~km} \mathrm{~s}^{-1}\right)\end{array}$ & $\begin{array}{c}V_{\text {mod }} \\
\left(\mathrm{km} \mathrm{s}^{-1}\right)\end{array}$ \\
\hline \multirow{23}{*}{ Group I } & 1 & -5.0 & 33.2 & $-84.3(0.4)$ & $-243.7(0.2)$ & $0.8(11)$ & $-11(7)$ & $11(7)$ \\
\hline & 2 & -5.7 & 7.1 & $-59.6(0.4)$ & $-242.9(0.2)$ & $14(14)$ & $-15(7)$ & $20(11)$ \\
\hline & 3 & -6.3 & 5.3 & $-191.1(0.4)$ & $-279.2(0.2)$ & $-9(13)$ & $16(7)$ & $18(9)$ \\
\hline & 4 & -7.8 & 1.1 & $-66.6(0.4)$ & $-237.8(0.2)$ & $5(14)$ & $-11(7)$ & $12(9)$ \\
\hline & 5 & -4.4 & 0.1 & $-57.9(0.3)$ & $-242.1(0.2)$ & & & \\
\hline & 6 & -3.1 & 0.6 & $-248.1(0.4)$ & $105.5(0.2)$ & $-9(13)$ & $8(7)$ & $12(11)$ \\
\hline & 7 & -2.7 & 1.1 & $-77.8(0.4)$ & $242.3(0.2)$ & $8(13)$ & $-10(7)$ & $13(10)$ \\
\hline & 8 & 0.1 & 0.5 & $-234.7(0.4)$ & $0.0(0.2)$ & $-9(13)$ & $2(7)$ & $9(13)$ \\
\hline & 9 & -0.2 & 0.6 & $-233.2(0.4)$ & $-0.4(0.2)$ & $-6(13)$ & $4(7)$ & $8(12)$ \\
\hline & 12 & -1.4 & 0.5 & $-186.7(0.4)$ & $-26.2(0.2)$ & $-26(16)$ & $9(8)$ & $28(16)$ \\
\hline & 13 & -2.2 & 0.3 & $-182.8(0.4)$ & $-27.4(0.2)$ & $0.9(13)$ & $9(7)$ & $9(7)$ \\
\hline & 14 & -1.3 & 5.2 & $-299.9(0.4)$ & $43.5(0.2)$ & $-13(13)$ & $5(7)$ & $14(13)$ \\
\hline & 15 & -1.7 & 0.7 & $-253.9(0.4)$ & $92.1(0.2)$ & $-7(13)$ & $6(7)$ & $9(11)$ \\
\hline & 16 & -1.9 & 0.3 & $-290.0(0.4)$ & $-24.0(0.2)$ & & & \\
\hline & 17 & -1.7 & 0.5 & $-281.9(0.4)$ & $-99.0(0.2)$ & $-6(14)$ & $-5(7)$ & $8(11)$ \\
\hline & 18 & 4.6 & 0.9 & $-171.4(0.4)$ & $-169.9(0.2)$ & $-18(14)$ & $4(7)$ & $18(13)$ \\
\hline & 19 & 1.9 & 0.5 & $-222.4(0.5)$ & $4.0(0.2)$ & $-0.3(16)$ & $11(8)$ & $11(8)$ \\
\hline & 20 & -2.0 & 0.1 & $-303.2(0.4)$ & $7.0(0.5)$ & $-12(13)$ & $-2(20)$ & $13(14)$ \\
\hline & 21 & -1.3 & 0.2 & $-184.5(0.4)$ & $-26(0.2)$ & $-18(13)$ & $16(7)$ & $24(11)$ \\
\hline & 22 & 0 & 0.3 & $-291.0(0.2)$ & $-5.8(0.1)$ & $-26(17)$ & $5(9)$ & $27(17)$ \\
\hline & 23 & 1.8 & 0.1 & $-290.1(0.3)$ & $-19.96(0.2)$ & & & \\
\hline & 24 & 1.3 & 0.1 & $-241.4(0.2)$ & $118.3(0.1)$ & $-5.0(17)$ & $3(9)$ & $6(15)$ \\
\hline & 25 & -0.6 & 0.1 & $-240.6(0.3)$ & $118.9(0.2)$ & & & \\
\hline \multirow{6}{*}{ Group II } & 10 & -0.1 & 0.7 & $199.8(0.4)$ & $89.2(0.2)$ & $22(14)$ & $11(7)$ & $24(13)$ \\
\hline & 11 & 0.9 & 0.1 & $197.4(0.4)$ & $90.6(0.2)$ & $6(13)$ & $19(7)$ & $20(8)$ \\
\hline & 26 & -7.4 & 6.6 & $-469.2(0.4)$ & $-1587.9(0.2)$ & & & \\
\hline & 27 & 4.1 & 0.5 & $-450.0(0.2)$ & $-1481.0(0.1)$ & & & \\
\hline & 28 & -5.7 & 4.9 & $-921.5(0.4)$ & $-1813.4(0.2)$ & $-17(14)$ & $-16(7)$ & $24(11)$ \\
\hline & 29 & -4.2 & 0.7 & $-921.6(0.4)$ & $-1812.9(0.2)$ & $-27(13)$ & $-18(7)$ & $32(12)$ \\
\hline
\end{tabular}

Note. For each identified feature, Col. 1 gives the label number; Cols. 2 and 3 the line-of-sight velocity and the integrated flux density of the highest-intensity channel; Cols. 4 and 5 the absolute positional (RA and Dec) offsets (and, within brackets, the associated errors), evaluated with respect to the maser position used at the correlator $\left(\alpha(\mathrm{J} 2000)=05^{\mathrm{h}} 30^{\mathrm{m}} 48^{\prime \prime}\right.$.033, $\delta(\mathrm{J} 2000)=33^{\circ} 47^{\prime} 54^{\prime \prime}$. 6$)$; Cols. 6-8 the projected components along the RA and Dec axes and the absolute value of the derived proper motions (and, within brackets, the associated errors).

integrated flux density increases with the $F W H M$ beam size of the observation, suggesting the presence of an extended component with size $>4^{\prime \prime}$ (the VLA-C beam). Going from 4.9 to $8.4 \mathrm{GHz}$ the VLA-C flux density increases by a factor of 2 (less than a factor of 3 , as expected for optically thick emission), whereas the VLA-D flux density remains largely constant. That is consistent with the presence of a compact core of emission at $4.9 \mathrm{GHz}$, that becomes optically thin at $8.4 \mathrm{GHz}$. Accordingly, our VLA-B measurements indicate that the integrated flux density remains constant or slightly decreases at frequencies higher than $8.4 \mathrm{GHz}$. Assuming an average optical depth $\approx 1$ at $8.4 \mathrm{GHz}$ for the emission inside the VLA-B beam $(\approx 0.9)$, one finds that the beam-averaged value for the density of the ionized gas is $2 \times 10^{5} \mathrm{~cm}^{-3}$.
Based on the extended nature of the radio continuum and the derived high value for the density of the ionized gas, the most likely interpretation is that of a young HII region. To account for the measured radio flux density, an ionising flux by a ZAMS star with spectral type B2 or earlier is required, corresponding to an expected value of stellar mass $\geq 10 M_{\odot}$ (Vacca et al. 1996; Palla et al. 2002).

\section{2. $22.2 \mathrm{GHz}$ water maser emission}

Figure 3 compares our VLA and VLBA results with previous interferometric observations. The top right-hand panels show the high-velocity molecular outflows seen in $\mathrm{HCO}^{+}(1 \rightarrow 0)$ and $\mathrm{SiO}(v=0,2 \rightarrow 1)$ with OVRO (Hunter et al. 1999). 

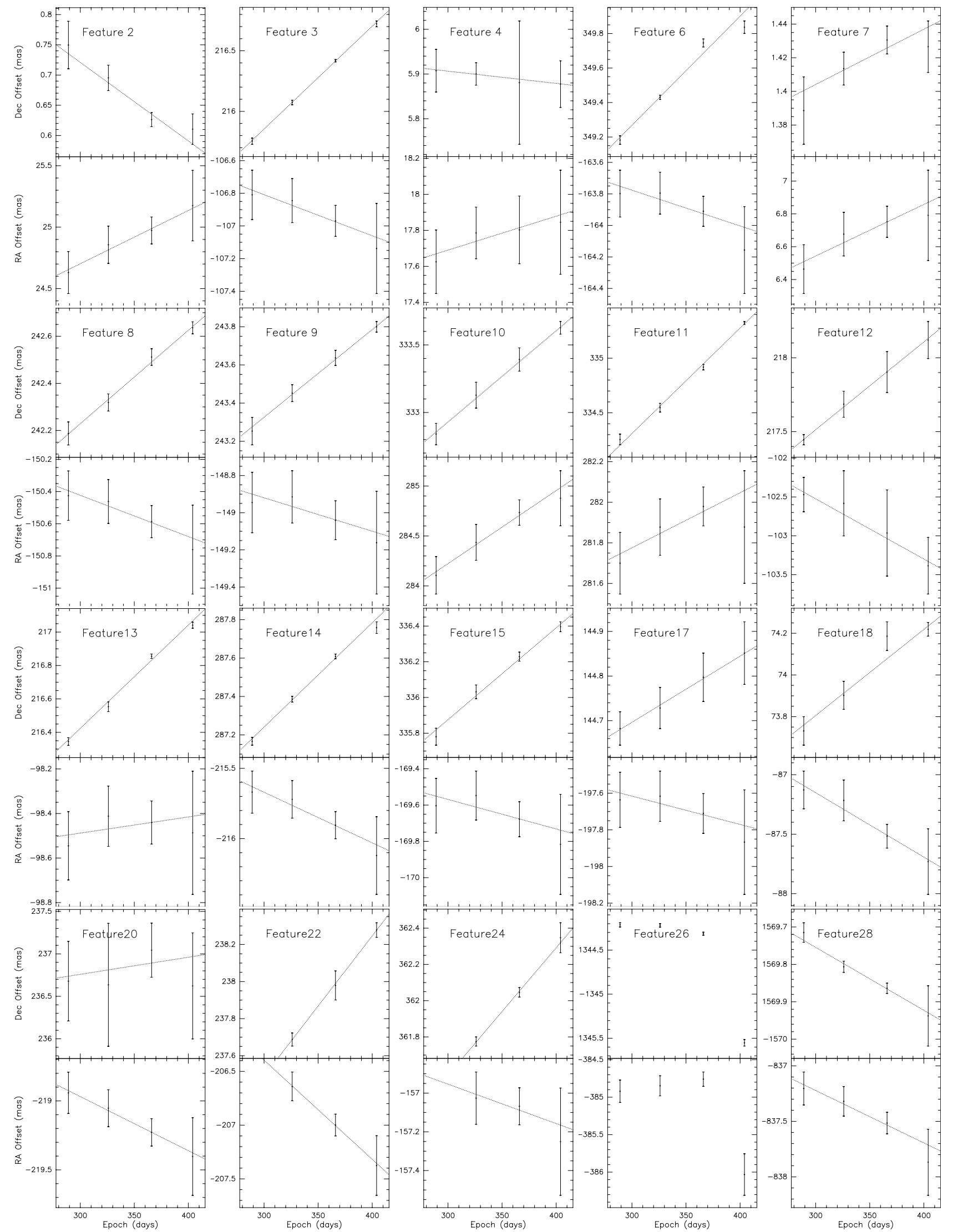

Fig. 1. Measured relative proper motions of the VLBA $\mathrm{H}_{2} \mathrm{O}$ maser features in AFGL 5142. For each of the time persistent features, the top and the bottom panels report the time variation of, respectively, the Dec and the RA offsets (relative to the reference feature "1"). In each panel, the dotted line shows the proper motion calculated by the (error-weighted) linear least-squares fit of the positional offsets with time. No proper motion is derived for the feature with label number " 26 ", since it does not show a linear variation of positional offsets with time. 


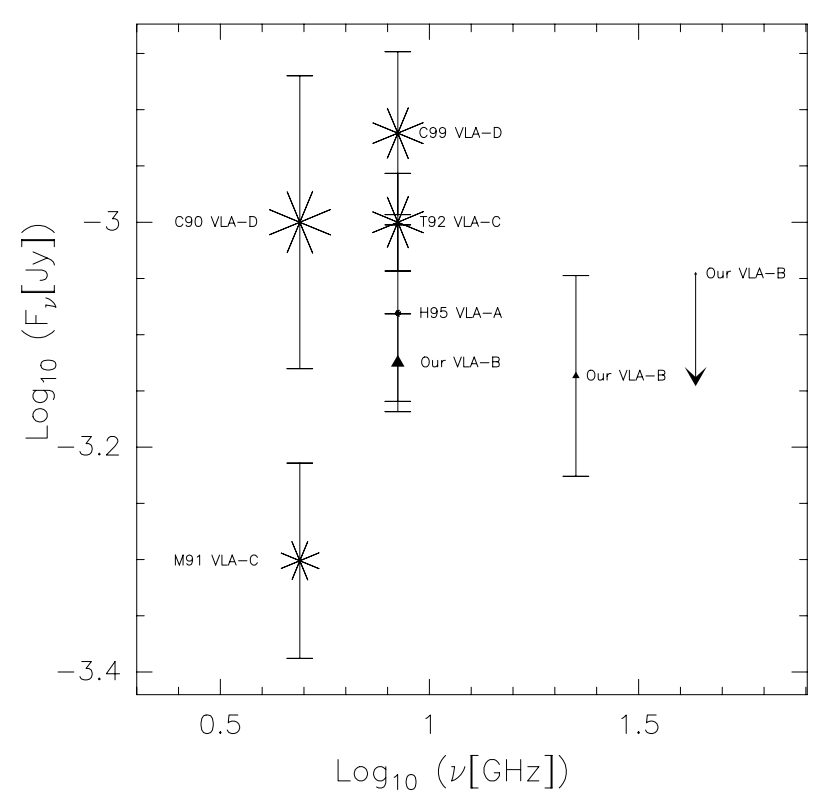

Fig. 2. The radio spectral energy distribution of AFGL 5142. Filled triangles are our VLA-B measurements of the flux density at 8.4 ( 0.75 mJy with a $\approx 0$ '. 9 beam), $22.4(0.7$ mJy with a $\approx 0$ '. 4 beam $)$, and $43.3 \mathrm{GHz}(\leq 0.9 \mathrm{mJy}$ with a $\approx 0$.' 2 beam; the upper limit is indicated by the downward arrow). The asterisks are previous flux density measurements at $4.9 \mathrm{GHz}(1.0 \mathrm{mJy}$ with $\mathrm{a} \approx 12.5$ beam using VLA-D Carpenter et al. 1990 (C90) - and 0.5 mJy with a $\approx 4$ " beam using VLA-C - McCutcheon et al. 1991 (M91)) and $8.4 \mathrm{GHz}(0.83 \mathrm{mJy}$ with $\mathrm{a} \approx 0$ '? 3 beam using VLA-A - Hunter et al. 1995 (H95); $1.0 \mathrm{mJy}$ with a $\approx 7$ '.7 beam using VLA-C - Torrelles et al. 1992 (T92); 1.2 mJy with a $\approx 7$ ". 5 beam using VLA-D - Carral et al. 1999 (C99)). The size of the symbols scales logarithmically with the FWHM size of the beam.

The $22.2 \mathrm{GHz}$ water masers in AFGL 5142 (prior of our EVN observations) were observed with the VLA at two epochs (1992, Hunter et al. 1995; 1998, Hunter et al. 1999), and are found within a few arcseconds from a compact, thermal continuum source (observed both with the VLA at $8.4 \mathrm{GHz}$ and with OVRO at $88 \mathrm{GHz}$ ), located at the center of the $\mathrm{SiO}-\mathrm{HCO}^{+}$outflow (Fig. 3, upper left-hand panel). The lower panel of Fig. 3 shows the positions and the velocities of the $\mathrm{H}_{2} \mathrm{O}$ maser features as derived by our multi-epoch VLBA observations, overlaid on our VLA map of the $22 \mathrm{GHz}$ continuum emission.

Based on the spatial distribution and the proper motion orientation of the detected maser features, the water maser emission appears to trace two elongated structures, oriented at quite different PA $\left(\triangle \mathrm{PA} \gtrsim 60^{\circ}\right)$. Using the same nomenclature as Paper I, we refer to such structures as Group I and Group II. In Table 3, we report the detected maser features separately for each Group.

Masers of Group I are found closer (within $\approx 300$ mas) to the $22 \mathrm{GHz}$ continuum emission peak. Based on the accurate absolute positions we have determined for the water masers and the thermal radio source, the $22 \mathrm{GHz}$ continuum peak falls at the center of the maser distribution of Group I (Fig. 3, lower panel). The features detected towards SE of the continuum source have LSR velocities blue-shifted (with respect to the LSR velocity of the region) whereas those detected towards NW are red-shifted. The measured transverse velocities of the blue and red-shifted features have similar amplitudes (in the range $6-27 \mathrm{~km} \mathrm{~s}^{-1}$ ) but opposite motion.

Maser features of Group II are concentrated in two clusters, having a sky-projected distance of $\sim 1700$ mas (corresponding to $\sim 3500 \mathrm{AU}$ at a distance of $1.8 \mathrm{kpc}$ ). The line connecting the two clusters is oriented along a northeast-southwest (NE-SW) direction and the average line-of-sight velocity of the features detected towards northeast is red-shifted whereas that of the southwestern features is blue-shifted. The measured proper motions indicate that the two clusters are moving away from each other along a NE-SW direction with similar velocities, in the range $20-32 \mathrm{~km} \mathrm{~s}^{-1}$.

In Fig. 3 (bottom panel), the line joining the two maser clusters of Group II does not cross the position of the VLA $22 \mathrm{GHz}$ continuum peak, but it is instead shifted S-E by $\gtrsim 0$.' 4 .

\section{Discussion}

The comparison of the spatial and velocity distribution of the molecular gas traced by the $\mathrm{HCO}^{+}$and $\mathrm{SiO}$ emission and the Group II water masers, suggests that these latter may be tracing the inner portion of the large-scale molecular outflow. This interpretation is based on three arguments: the elongated distribution of the masers is almost parallel to the position angle of the $\mathrm{HCO}^{+}$outflow; the blue-shifted features lie northward and the red-shifted ones lie southward, qualitatively matching the velocity distribution of the large-scale molecular outflow; the orientation of the measured proper motions is approximately aligned with the axis of the molecular outflow.

In Paper I, based on low-sensitive EVN data, the observed velocity pattern of Group I masers has been interpreted in terms of Keplerian rotation. In order to test the Keplerian hypothesis, we fitted the positions and the 3D absolute velocities of maser features with the Keplerian disk model described in Paper I (see Appendix A for a more detailed description of the model formalism). The minimizing algorithm (Eq. (A.1) in Appendix A) converged to a best fit solution. The geometrical parameters of the best fit disk are found to be in good agreement with those previously derived with the EVN maser data (see Paper I). Since the measured relative velocities are in our case three-five times more accurate than absolute velocities (see Sect. 2.2.5), we repeated the fit comparing model predicted and measured relative (to the reference maser feature) velocities (Eq. (A.2) in Appendix A). In this case, the Keplerian model failed to find a minimum, indicating that the measured velocity field for the Group I masers is actually incompatible with Keplerian rotation. This result demonstrates that the solution obtained fitting both absolute (VLBA) and relative (EVN) proper motions is a spurious result owing to the large measurement errors.

The lack of success of the Keplerian model is not surprising. Looking at the Fig. 3, one notes that the motion traced by Group I water masers appears to be expansion from (rather than rotation around) the putative location of the YSO, as marked by the peak of the thermal continuum emission. In particular, proper motions in both Group I (SE and NW) maser clusters are oriented outward from the expected YSO position, whereas in a Keplerian rotation they should be directed both inward and outward, tracing both the far and near side of a disk annulus. 


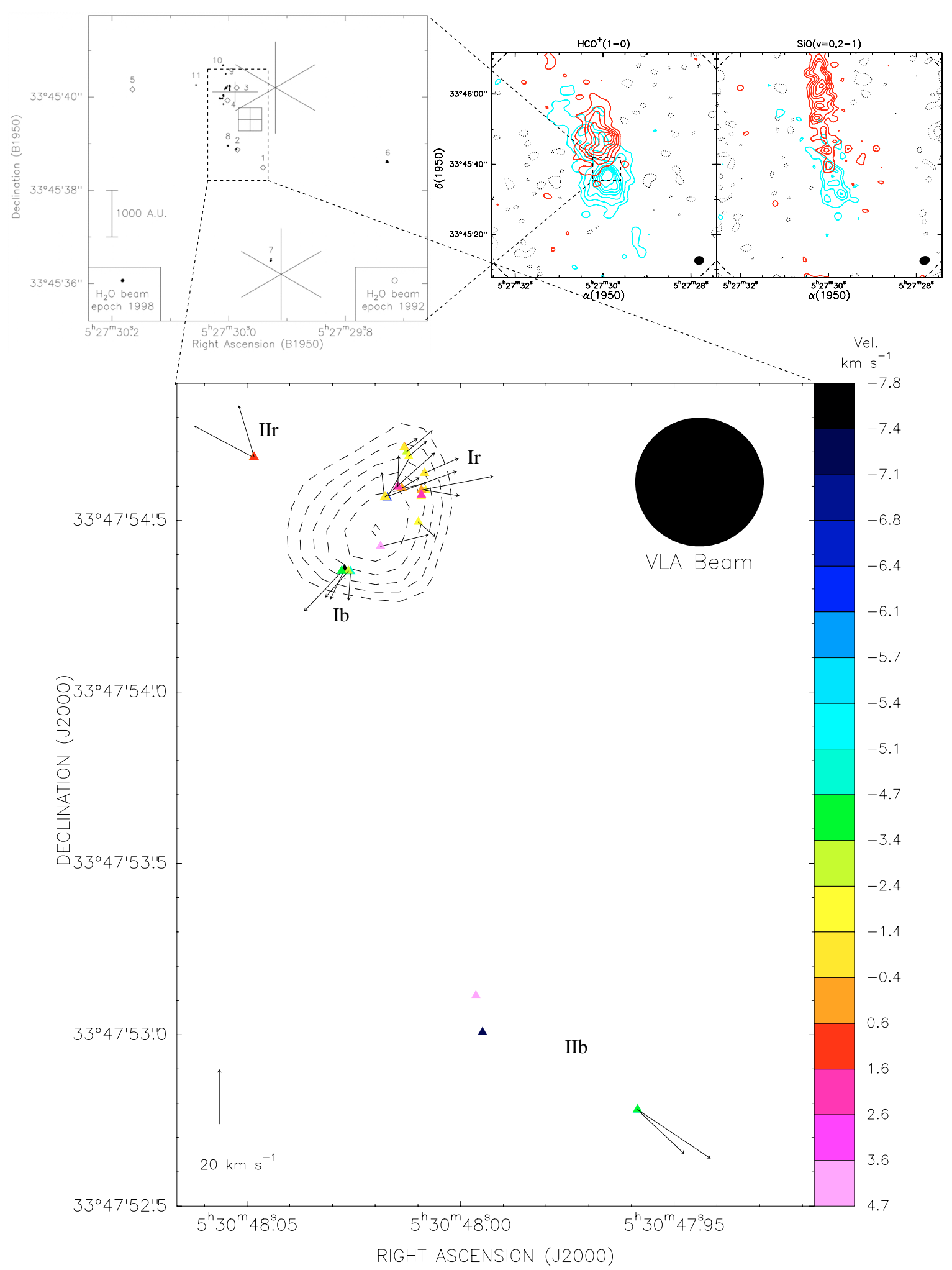

Fig. 3. VLA and VLBA results towards AFGL 5142. Upper right-hand panel: contour maps of the OVRO high-velocity emission of $\mathrm{HCO}^{+}$ $(1 \rightarrow 0)$ (left) and $\mathrm{SiO}(v=0,2 \rightarrow 1)$ (right) (Hunter et al. 1999). Upper left-hand panel: positions of the $22 \mathrm{GHz}$ masers detected with the VLA in 1992 (open squares) and 1998 (filled dots); OVRO $88 \mathrm{GHz}$ (boxed cross) and VLA $8.4 \mathrm{GHz}$ (plain cross) continuum sources (Hunter et al. 1995, 1999). Bottom panel: positions of the VLBA $\mathrm{H}_{2} \mathrm{O}$ maser features are marked with filled triangles, different colours denote the features' LSR velocities, according to the colour scale on the right-hand side of the panel, and the arrows indicate the measured absolute proper motions, whose amplitude scale is given at the bottom of the panel; the labels Ib, Ir and IIb, IIr individuate the blue-shifted and red-shifted maser clusters

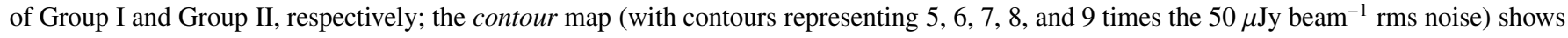
the $22 \mathrm{GHz}$ VLA continuum emission; the filled circle in the upper right corner represents the restoring beam of the VLA-B $22 \mathrm{GHz}$ continuum map. 
To test if Group I masers trace an outflowing motion, the data were fitted with the conical outflow model described in detail in Goddi et al. (2005). Since the absolute values of the measured velocities do not present a large variation (compared with the observational errors - see Table 3), in the conical outflow model we assumed the maser features to move with a constant velocity (differently from the Hubble motion assumption in Goddi et al. 2005). The best fit solution was looked for minimizing the $\chi^{2}$ given by the sum of the square differences between the model and measured, relative, $3 \mathrm{D}$ velocities (Eq. (A.2) in Appendix A). The fit parameters were searched for over a wide range of physically plausible values: the cone vertex (i.e., the YSO location) within a distance of $300 \mathrm{AU}$ from the $22 \mathrm{GHz}$ continuum peak; the cone axis orientation over the full $4 \pi$ solid angle; the cone aperture within the interval $10-85^{\circ}$; the constant expansion velocity from 1 to $50 \mathrm{~km} \mathrm{~s}^{-1}$. The fit parameters were varied with steps comparable to or smaller than the associated fit errors. The minimization algorithm converged towards the best fit solution. The best fit conical jet is found to have a large $\left(\approx 80^{\circ}\right)$ opening angle. The best fit cone-axis is predicted to form an angle of $\approx 30^{\circ}$ with the line-of-sight and to have a position angle projected onto the plane of the sky of $\approx 28^{\circ}$. The (constant) expansion velocity has an absolute value, $v_{0}$, of $13 \mathrm{~km} \mathrm{~s}^{-1}$.

At this point a fundamental question arises: what might be the driving source of the observed flow motions of Group I and II masers?

First we consider the scenario where a unique YSO is responsible for the radio continuum emission, the excitation of both maser groups, and the acceleration of the large scale molecular outflow. Since Group I shows a dense $\left(n_{\mathrm{H}_{2}}>\right.$ $10^{7} \mathrm{~cm}^{-3}$ - Elitzur et al. 1989; Kaufman \& Neufeld 1996) clump of gas around the expected YSO position, oriented on the sky at a large angle from the axis of the jet/outflow system traced by the Group II maser and (on a larger scale) by the $\mathrm{HCO}^{+}-\mathrm{SiO}$ emission, Group I masers may originate in the accretion disk surrounding the YSO. We speculate that Group I maser features might emerge from the atmosphere of a flared disk (with a disk scale height $H(r)$ scaling linearly with $r$ ), in the region where modern MHD models predict the launch of the protostellar jet. As stressed in Sect. 1, such models can be divided into two categories, $\mathrm{X}$-wind and disk-wind. In the former model, the jet is expected to originate within $0.1 \mathrm{AU}$ of the YSO and to collimate at radii $\leq 10 \mathrm{AU}$, whereas the latter model predicts the wind to be launched across a significant range of disk radii (from tens of $\mathrm{AU}$ up to several hundreds of $\mathrm{AU}$, for high-mass YSOs) and to collimate along the disk axis at correspondingly larger distances from the YSO. The detected $\mathrm{H}_{2} \mathrm{O}$ masers are distributed at distances from the YSO position in the range 10-600 AU. These large distances are clearly incompatible with the $\mathrm{X}$-wind requirements, but they might still find an explanation within the disk-wind scenario. In this view, the disk-wind model would explain both Group I and Group II water masers, these latter tracing the collimated portion of the jetoutflow system at larger distances from the YSO (>1000 AU).

In the proposed scenario of a single powering source, one would expect the NE-SW outflow axis to be centered in between the maser clusters Ir and Ib, i.e. at the position of the continuum source. That is not the case and this is a clear difficulty for the disk-wind interpretation.

The alternative scenario is the one where the water masers in AFGL 5142 trace two physically uncorrelated jets (one directed towards SW-NE and the other one towards SE-NW), each jet being powered by a distinct YSO. As already discussed in Sect. 3.1, the YSO responsible for the excitation of both the ionized source and the Group I masers is expected to be massive $\left(\geq 10 M_{\odot}\right)$. The other YSO should power the Group II masers and the $\mathrm{HCO}^{+}-\mathrm{SiO}$ outflow, which contains a mass of $35 M_{\odot}$ (Hunter et al. 1999). From excitation and energetic considerations one would expect this YSO to be also a massive one. Geometrical considerations also suggest that this YSO may be located on the axis of the outflow traced by the Group II masers. Along this axis, separated by more than 0.' 4 from the $22 \mathrm{GHz}$ continuum peak, no compact continuum emission was detected by our sensitive VLA observations. A possible explanation is that the YSO exciting Group II masers might be found in an evolutionary stage earlier than the YSO powering the Group I masers, when the ionized gas is still too confined by the dense circumstellar environment to be detectable.

We have reviewed the literature looking for indications of a multiple system of YSOs in the region of detection of the water masers. Hunter et al. (1995) and, very recently, Chen et al. (2005) performed NIR $J H K$ and $\mathrm{H}_{2} v=1-0 S$ (1) imaging observations of the molecular cloud core in AFGL 5142, revealing the presence of a cluster of YSOs distributed over a region of diameter of a few arcminutes. $\mathrm{H}_{2} \mathrm{O}$ masers are found corresponding to the infrared source NIRS1 of Chen et al. (2005) (IRS1 in the notation of Hunter et al. 1995). The minimum distance between NIRS1 and another member of the cluster is $\gtrsim 5^{\prime \prime}$ (see Table 2 of Chen et al. 2005), whereas (considering the diameter of the maser distribution) the two (putative) YSOs powering the two maser groups should be separated by a distance 0. . $4 \lesssim d \lesssim 2^{\prime \prime}$ (corresponding to $700 \lesssim d \lesssim 3600 \mathrm{AU}$ ). Hence, the available NIR observations, having an angular resolution $\gtrsim 1^{\prime \prime}$, do not allow us to identify two distinct powering sources for maser Groups I and II. Towards AFGL 5142, considering an angular scale larger than that presented in Fig. 3 (top right-hand panels), a complex pattern of molecular outflows has also been found, which might suggest the presence of a multiple YSO system: Hunter et al. (1995) detected two CO outflows, nearly perpendicular to each other, one, directed towards SE-NW, extended on scales of a few arcminutes, the other one, directed towards SW-NE, more compact; Hunter et al. (1999) detected a $\mathrm{HCO}^{+}-\mathrm{SiO}$ outflow elongated in a N-S direction (Fig. 3, upper right-hand panels); Chen et al. (2005) found $\mathrm{H}_{2}$ jet-like structures along each of the three molecular outflows. Chen et al. (2005) propose that the SW-NE oriented jet/outflow system is powered by NIRS1, whereas the SE-NW oriented jet/outflow system is driven by NIRS30, 30" apart from NIRS1 (corresponding to the IRAS source in the AFGL 5142 SFR). Since NIRS1 and NIRS30 are now the only two recognized massive YSOs of the AFGL 5142 cluster (Chen et al. 2005), the available molecular observations (with an angular resolution $\gtrsim 1^{\prime \prime}$ ) provide no hints for the presence of two massive YSOs within the region of a few arcsec where the water maser emission is observed. 
Thus, even if the geometry of the outflows traced by the two detected maser Groups may favour the interpretation of two distinct exciting YSOs, the data presently available do not permit us unambiguously to identify more than a single YSO in the region of maser emission. In this respect, the axes of the outflows traced by water masers on scales of hundreds and thousands of AU can be good places to look for the YSOs and the present water maser observations can drive future studies of the SFR AFGL 5142.

\section{Conclusions}

This article reports the results of VLBA multi-epoch observations of the $22.2 \mathrm{GHz}$ water masers and a VLA multi-frequency study of the continuum emission towards the SFR AFGL 5142. With respect to the previous EVN observations, the VLBA provided a significant improvement in sensitivity (by a factor $\sim 10$ ) and an optimized time separation between consecutive epochs ( 1 month vs. 3-6 months), resulting in a larger number of detected maser features (29 vs. 12) and measured proper motions (over three or four epochs, 23 vs. 2). The VLBA observations were performed in phase-reference mode, allowing the determination of absolute positions and proper motions of the water maser features.

The water maser emission is found to originate from two elongated structures, with the measured proper motions aligned along the structures' elongation axes. Using the same nomenclature as Paper I, we refer to such structures as Group I and Group II. Each group consists of two (blue- and red-shifted) clusters of features separated by a few hundred AU for Group I and thousands of AU for Group II.

We detect a compact radio continuum source at 8.4 and $22 \mathrm{GHz}$ (previously reported at 4.9 and $8.4 \mathrm{GHz}$ ) located exactly at the center of the Group I maser distribution, indicating that the source ionizing the gas is also responsible for the excitation of the water masers. Comparing our VLA-B observations with previous VLA-C and VLA-D data, there is an indication that the continuum emission is extended on angular scales $>4^{\prime \prime}$ and is optically thin at frequencies higher than $8.4 \mathrm{GHz}$. The most likely interpretation is in terms of a young HII region.

The measured transverse velocities of maser features indicate that the two clusters of Group II are moving away from each other along the cluster-connecting line. The spatial and velocity distribution suggests that the water masers of Group II trace a collimated outflow. Since the axis of the larger-scale $\mathrm{HCO}^{+}-\mathrm{SiO}$ outflow is almost parallel to the direction of elongation and motion of the Group II maser features, these latter likely trace the inner portion of the outflow and originate in dense clumps displaced along the outflow axis.

Group I maser features indicate a clump of dense gas around the expected YSO position (pinpointed by the VLA continuum) and the measured proper motions appear to trace expansion. If a single YSO were responsible for the excitation of both maser groups, the proposed disk-wind scenario might explain the observed flow motion of both Group I masers, tracing the dense material emerging from the disk atmosphere, and the Group II maser jet, corresponding to the portion of the wind collimated along the disk-axis at larger distances from the
YSO. Since the axes joining the two clusters of maser Groups I and II do not intersect at the position of the continuum location, we favour the scenario where two distinct massive YSOs are driving the two maser outflows. Presently, the available observational data, from radio to NIR wavelengths, do not permit us to identify unambiguously more than a single massive YSO in the region of the detected maser emission. Our detection of the maser outflows can drive future studies to localize the powering YSOs.

Acknowledgements. We thank the anonymous referee for pointing out the presence of previous observations indicating the extended nature of the continuum emission.

\section{Appendix A: Kinematical model formalism}

To investigate the kinematical structure traced by Group I water masers, we fitted the positions and the 3D (line-of-sight + transverse) velocities of maser features with two simple alternative kinematical models: a Keplerian disk and a conical outflow. The model fitting was performed using both measured absolute and relative velocities.

\section{A.1. Fit of the absolute velocities}

The best-fit solution is found minimising the $\chi^{2}$ given by the sum of the square differences between the model and measured, absolute, 3D velocities:

$\chi^{2}=\sum_{i=1}^{n s p o t}\left[\frac{v_{z}^{i}-\left(V_{z}^{i}-V_{\text {cloud }}\right)}{\Delta V_{z}^{i}}\right]^{2}+\sum_{k} \sum_{j: x, y}\left[\frac{v_{j}^{k}-V_{j}^{k}}{\Delta V_{j}^{k}}\right]^{2}$

where the lowercase $v$ denotes the velocity component computed with the model and the uppercase $V$ is the measured velocity component, with the corresponding uncertainty given by $\Delta V$. In the first term of the sum, only the line-of-sight velocities (along the " $z$ " axis) of the identified features (indicated with the index $i$ ) are considered. In order to compare with the model, the measured line-of-sight velocities have been first corrected by the peculiar motion of the SFR $\left(V_{\text {cloud }}=-4.4 \mathrm{~km} \mathrm{~s}^{-1}\right.$, as deduced by CO emission - Snell et al. 1988), implicitly assuming the LSR velocity of the (proto-)star to be the same as the surrounding molecular cloud. The uncertainty of the line-of-sight velocities is taken equal to the spectral FWHM of the maser line, $\lesssim 1 \mathrm{~km} \mathrm{~s}^{-1}$. The second term of the sum includes the features (indicated with the index $k$ ) with measured transversal velocities, whose components along the RA (" $x$ ") and Dec (" $y$ ") axes are denoted with the index $j$. In the model, the transversal velocity of the YSO is taken to be zero, so that we compared directly the model and the measured absolute transversal velocities.

\section{A.2. Fit of the relative velocities}

Fitting absolute velocities has two disadvantages: 1) the absolute motions have large errors (see Col. 8 of Table 3); 2) the model assumption that the YSO does not move across the plane 
of the sky may be wrong. In order to overcome these limitations, the fit was repeated comparing the model predicted and measured relative (to the reference maser feature) velocities:

$$
\begin{aligned}
\chi^{2}= & \sum_{i=1}^{n s p o t}\left[\frac{\left(v_{z}^{i}-v_{z}^{r}\right)-\left(V_{z}^{i}-V_{z}^{r}\right)}{\Delta V_{z}^{i}}\right]^{2} \\
& +\sum_{k} \sum_{j: x, y}\left[\frac{\left(v_{j}^{k}-v_{j}^{r}\right)-V_{j}^{k}}{\Delta V_{j}^{k}}\right]^{2}
\end{aligned}
$$

where the $V_{j}^{k}$ are the measured relative proper motions, $\Delta V_{j}^{k}$ are the associated uncertainties (within a few $\mathrm{km} \mathrm{s}^{-1}$ in all cases) and the index $r$ indicates the reference maser feature. Comparing the model and observed relative velocities has the advantage not to require the inclusion as a further model free parameter the velocity of the YSO.

\section{References}

Bonnell, I. A., \& Bate, M. R. 2002, MNRAS, 336, 659

Carpenter, J. M., Snell, R. L., \& Schloerb, F. P. 1990, ApJ, 362, 147

Carral, P., Kurtz, S., Rodríguez, L. F., et al. 1999, Rev. Mex. Astron. Astrofis., 35, 97
Chen, Y., Yao, Y., Yang, J., Zeng, Q., \& Sato, S. 2005, ApJ, 629, 288

Elitzur, M., Hollenbach, D. J., \& McKee, C. F. 1989, ApJ, 346, 983

Goddi, C., Moscadelli, L., Alef, W., \& Brand, J. 2004, A\&A, 420, 929

Goddi, C., Moscadelli, L., Alef, W., et al. 2005, A\&A, 432, 161

Hunter, T., Testi, L., Taylor, G., et al. 1995, A\&A, 302, 249

Hunter, T., Testi, L., Zhang, Q., \& Sridharan, T. 1999, AJ, 118, 477

Imai, H., Kameya, O., Sasao, T., et al. 2000, ApJ, 538, 751

Kaufman, M. J., \& Neufeld, D. A. 1996, ApJ, 456, 250

Konigl, A., \& Pudritz, R. E. 2000, Protostars and Planets IV, 759

McCutcheon, W. H., Dewdney, P. E., Purton, C. R., \& Sato, T. 1991, AJ, 101, 1435

Moscadelli, L., Cesaroni, R., \& Rioja, M. J. 2005, A\&A, 438, 889

Palla, F., Zinnecker, H., Maeder, A., \& Meynet, G. 2002, Physics of star formation in galaxies

Seth, A., Greenhill, L. J., \& Holder, B. P. 2002, ApJ, 581, 325

Shu, F. H., Adams, F. C., \& Lizano, S. 1987, ARA\&A, 25, 23

Shu, F. H., Najita, J. R., Shang, H., \& Li, Z.-Y. 2000, Protostars and Planets IV, 789

Snell, R. L., Huang, Y.-L., Dickman, R. L., \& Claussen, M. J. 1988, ApJ, 325, 853

Torrelles, J. M., Gomez, J. F., Anglada, G., et al. 1992, ApJ, 392, 616

Torrelles, J. M., Patel, N. A., Anglada, G., et al. 2003, ApJ, 598, L115

Vacca, W. D., Garmany, C. D., \& Shull, J. M. 1996, ApJ, 460, 914

Yorke, H. W., \& Sonnhalter, C. 2002, ApJ, 569, 846 